\title{
Prevalência de hepatite C em pacientes Com lúpus ERITEMATOSO SISTÊMICO DO HUCCF-UFRJ
}

\author{
Cláudia de Abreu Costa*, José Angelo Papi, Henrique Sérgio de M. Coelho, \\ Carmem Nogueira, Bart Vanderboght, Jorge André de S. Soares \\ Trabalho realizado no Hospital Universitário Clementino Fraga Filho-Universidade Federal do Rio de Janeiro, RJ
}

RESUMO - Os pacientes com lúpus eritematoso sistêmico (LES) apresentam fatores de risco para a aquisição de hepatite $\mathrm{C}$, como hospitalizações e hemotransfusões, e compartillham com os pacientes infectados pelo vírus da hepatite $\mathrm{C}(\mathrm{HCV})$ várias manifestações clínico-laboratoriais relacionadas a auto-imunidade.

OBjEtivo. 0 objetivo do presente estudo é determinar a prevalência de hepatite $\mathrm{C}$ em uma população com LES acompanhada no ambulatório de programas especiais - Colagenoses do Hospital Universitário Clementino Fraga Filho (HUCFF. UFRJ).

Métodos. Foi realizado um estudo de corte-transversal, incluindo os primeiros 91 pacientes com o diagnóstico de LES provável ou definitivo procedentes do referido ambulatório, no período de abril a setembro de 1997. Todos os doentes foram avaliados, sob o ponto de vista clínico e laboratorial, para determinação de atividade de doença lúpica. Foram realizados o teste de triagem UBI HCV EIA 4.0 e 0 teste suplementar INNO-LIA HCV AbIII nos 91 pacientes selecionados e, em 32 pacientes escolhidos ao acaso e nos pacientes reativos aos testes imunossorológicos foi realizada a pesquisa do RNA-HCV pelo método da reação de polimerização em cadeia (PCR).

Resultados. Observou-se um aumento significativo da prevalência de anti-HCV na amostra, quando comparada à população de doadores de sangue do HUCFF $(6,6 \%$ versus $1,39 \%$ com 0 teste EIA, valor $p=0,02$ e intervalo de confiança $95 \%=5,5$ a $13,8)$, contudo, considerando-se resultados obtidos com os testes confirmatórios imunoblot e $P C R$ aplicados em série (prevalência de HCV de 2,2\%, com intervalo de confiança $95 \%=0,2$ a 7,7 ) , a prevalência de HCV em pacientes com LES não parece mais alta que em doadores de sangue.

Conclusōes. A prevalência de hepatite $\mathrm{C}$ na amostra estudada utilizando-se um teste de triagem (EIA) foi significativamente maior do que a de uma população de doadores de sangue $(6,6 \%$ versus $1,39 \%$ ). Porém, aplicando-se em série os testes confirmatórios INNO-LIA ${ }^{\circledR}$ e PCR (prevalência de hepatite C de 2,2\%) parece não haver evidências de que pacientes com LES tenham maior risco de adquirir o HCV do que a população em geral, embora a prevalência tende a ser maior que a população de doadores de sangue. Em pacientes com diagnóstico provável de LES deve-se avaliar o custo/benefício de se investigar HCV e crioglobulinemia mista essencial (CME).

Unitermos: Lúpus eritematoso sistêmico. Hepatite C. Autoimunidade.

\section{INTRODUÇÃO}

Desde a sua descoberta, em 1989, muitos aspectos clínicos extra-hepáticos e anormalidades imunológicas têm sido relacionados com a infecção crônica pelo HCV?. À semelhança com os pacientes com LES, pacientes com hepatite $C$ crônica apresentam freqüentes queixas musculoesque-

\footnotetext{
*Correspondência:

Cláudia de Abreu Costa

Rua Barão de São Francisco, 120 - apto. 802 -

Andaraí - Fone: 20560-030 - Rio de Janeiro - RJ e-mail: jfclaudia@uol.com.br - Tel.: (21) 2268-6936
}

léticas 13,23,27,33,43,45. O quadro clínico, relatado por vários autores, descreve os pacientes com RNA-HCV circulante com artralgias e poliartrite cumulativa e não-erosiva, alguns inclusive respondem a baixas doses de corticóide, como no LES ${ }^{13,23,43}$. Trombocitopenia, um dos critérios diagnósticos maiores do LES, foi também observada nos pacientes com HCV28,38.

Crioglobulinemia mista essencial, com manifestações de vasculite cutânea, glomerulonefrite ou neuropatia, foi observada em ambas as doenças com fre- qüências amplamente varáveis, 2,8,24,26,30,44

Associado à crioglobulinemia, os pacientes infectados pelo HCV podem apresentar também hipocomplementemia, presença de fator reumatóide e de anticorpos antinucleares (ANA) $)^{7,10,16,31,32,45}$. Estes mesmos achados são geralmente observados nas fases de atividade de doença do LES36.

Diversos fatores genéticos, ambientais, hormonais, têm sido implicados para justificar o polimorfismo de apresentação clínica e evolução do LES. Evidências clínicas e laboratoriais sugerem que infecções 
virais persistentes poderiam levar a uma ativação policlonal sustentada das células $B$ nos indivíduos com predisposição genética para tal. Desta maneira, o próprio HCV poderia facilitar o surgimento ou modificar a história natural do LES 3.

Éimportante determinar a freqüência da associação entre LES e infecção pelo HCV, devido a implicações clínicas e terapêuticas. Descreveram-se casos de indução e exacerbação das manifestações clínicas do LES após tratamento com Interferon para hepatite e, por outro lado, o comportamento biológico do HCV frente a uma doença da auto-imunidade generalizada como LES ainda não é conhecido 1,29,41.

Além disso, é reconhecido que os pacientes com LES apresentam fatores séricos que podem interferir nos resultados dos testes imunosorológicos, tais como fator reumatóide, hipergamaglobulinemia e complexos imunes circulantes, deste modo podendo contar com resultados falso-positivos $\$ 8,40$.

\section{Métodos}

Delineamento do estudo: estudo de corte transversal, no período de abril// 997 a setembro/1997, aprovado pela Comissão de Ética do HUCFF no mesmo ano. Critérios de inclusão e exclusão: Foram incluídos no estudo 95 pacientes com diagnóstico definitivo ou provável de LES segundo os critérios de classificação do Colégio Americano de Reumatologia $(A C R)^{38}$.

Foi previamente determinado que seriam incluídos no estudo os primeiros 95 pacientes que comparecessem ao laboratório de pesquisa em Biologia Molecular, independentemente da idade, sexo, gravidade de doença, ou tempo prévio da mesma. Eles eram convidados a participarem do estudo durante a consulta ambulatorial com seus respectivos médicos e, após consentimento verbal, eram encami- nhados ao laboratório, onde eram realizados os exames da rotina do ambulatório e incluídos os testes sorológicos para HCV. Foram excluídos do estudo pacientes com o diagnóstico de LES induzido por drogas e aqueles que após revisão dos prontuários não preencheram os três critérios diagnósticos mínimos determinados pela ACR (quatro pacientes entre os 95 selecionados inicialmente).

\section{Análise clínica da amostra}

Os pacientes foram entrevistados segundo os dados contidos em ficha preestabelecida, caracterizada por dados demográficos, tempo de doença lúpica, doenças associadas, uso de medicamentos modificadores da resposta imune e tempo de uso dos mesmos. Avaliou-se a presença de fatores de risco reconhecidos para infecção pelo $\mathrm{HCV}^{3,41}$. Dados clínicos sugestivos de comprometimento dos diversos sistemas orgânicos foram também pesquisados para avaliação da atividade de doença lúpica ${ }^{5}$.

\section{Análise laboratorial}

Exames hematológicos, bioquímicos e sorológicos de rotina para avaliação de atividade de doença lúpica foram realizados na amostra nos 95 pacientes com LES selecionados inicialmente

Avaliação do índice de atividade de doença SLEDAI (Systemic Lupus Erythematosus Disease Activity Index) 5, 14, 15,22,37.

A pontuação do índice SLEDAI foi realizada para avaliar uniformemente todos os pacientes da amostra quanto à atividade de doença.

\section{Testes sorológicos para hepatite $\mathbf{C}$}

Para detecção dos anticorpos para o HCV foi utilizado o teste UBI HCV EIA 4.0 $\left(\mathrm{ORGANON}^{\circledR}\right)^{4,35}$, um imunoensaio enzimático qualitativo. A amostra foi considerada reativa quando a densidade ótica média foi igual ou superior a 20\% do valor do cut-off. $O$ teste tipo immunoblot foi aplicado em todos os pacientes para excluir resultados falsos-positivos, tendo sido utilizado o teste INNO-LIA HCV Ab III ${ }^{\circledR}$ (INNOGENETICS ${ }^{\circledR}$, Bélgica). A amostra foi considerada reativa. Realizou-se também a pesquisa de HCV-RNA pelo método qualitativo - PCR (Polymerase Chain Reaction) em 38 amostras de soro. A escolha das amostras foi aleatória. Os primersutilizados para a pesquisa do RNAHCV foram os mesmos usados no teste de genotipagem do vírus C - InnoLiPA HCV II ${ }^{\circledR}$ (INNOGENETICS ${ }^{\circledR}$, Bélgica).

\section{População de comparação}

O grupo de comparação para este estudo foi a população de doadores de sangue do HUCFF. No período de primeiro de janeiro a 31 dezembro de 1997 foi determinado a prevalência de anti-HCV + pelo método EIA (UBI HCV EIA 4.0) nos candidatos a doação de sangue aprovados na entrevista de triagem no banco de sangue do HUCFF neste período.

\section{Análise estatística}

Realizada análise descritiva das características demográficas, clínicas, e laboratoriais da amostra. Com o auxílio estatístico do programa de informática Epilnfo versão 6,03, calculou-se a prevalência de anti-HCV + na amostra para cada um dos métodos imunossorológicos testados e quando os testes (EIA e INNO-LIA HCV AbIII ${ }^{\circledR}$ ) foram utilizados em série ${ }^{12}$. Calculou-se o intervalo de confiança de 95\% (pela exata binomial) da prevalência de anti-HCV+ encontrada nos testes isoladamente ou em série e também determinou-se a razão de prevalência quando comparou-se as prevalências de anti-HCV entre os doadores de sangue e a amostra estudada. Utilizou-se o coeficiente Kappa como medida de confiabilidade entre os testes imunossorológicos empregado (EIA $3^{\text {a }}$ geração e INNO-LIA ${ }^{\circledR}$ ). 


\section{Resultados}

\section{Características da amostra}

Dos pacientes, 84 (92\%) eram do sexo feminino e sete (8\%) do sexo masculino A média de idade da amostrafoide 40 anos +1 - II,86 e um intervalo variando de 14 a 68 anos. A média do tempo de duração da doença lúpica foi de 7,8 anos; com um desvio-padrão amplo (7,9 anos ) e uma variação entre menos que I ano a 43 anos.

Os pacientes foram estratificados em três grupos, de acordo com o tempo de duração do LES: 9\% dos pacientes apresentaram menos de I ano do diagnóstico, em 37\% o tempo de duração do LES encontrou-se entre I e 5 anos e $53 \%$ dos pacientes apresentaram mais de 5 anos de evolução da doença.

A média da pontuação total do SLEDAl foi de 6 (desvio-padrão de 5,76), com uma variação entre 0 e 28 . De 0 a 5 na pontuação foram encontrados $43 \%$ dos pacientes, entre 5 e 10 pontos foram agrupados $32 \%$ dos pacientes, e os restantes $25 \%$ apresentavam uma pontuação superior a 10 , indicando atividade inflamatória grave.

No momento do estudo, $72 \%$ estavam em uso de doses variáveis de prednisona, $68 \%$ estavam em uso de cloroquina, 22\% apresentavam-se em pulsoterapia mensal e trimestral com ciclofosfamida IV e $4 \%$ faziam uso de talidomida. Não faziam uso de nenhum medicamento modificador da resposta imune apenas 10\% dos pacientes da amostra.

Os pacientes com LES estudados apresentaram alguns fatores de risco reconhecidos para hepatite $C^{3,41}$ : I5\% foram hemotransfundidos em algum momento de sua vida, $26 \%$ foram submetidos a mais de uma hospitalização e 12\% foram submetidos a ambos procedimentos. Em 60 pacientes estudados na amostra não foram identificados fatores de risco para aquisição da hepatite C (65,9\%); nenhum paciente se encon- trava em tratamento hemodialítico.

Anormalidades

nas enzimas ALT e AST foram evidenciadas, respectivamente, em apenas 3 e 5 dos 91 pacientes, sendo que uma das pacientes com elevação de ambas as enzimas apresentava critérios clínicos e histopatológicos para miosite grave, sendo bastante provável que tais achados laboratoriais fossem secundários ao processo inflamatório muscular, e não hepático.

\section{Prevalência de Hepatite $C$ na amostra}

A prevalência de anti-HCV+ pelo EIA foi de 6,6\% (com IC95\% = 5,5 a 13,8), enquanto pelo INNO-LIA ${ }^{\circledR}$ foi de $4,4 \%$ (com IC95\%=I,2 a I0,9). A prevalência de reatividade ao anti-HCV na população de comparação de doadores de sangue foi fornecida pelo Relatório Anual do Banco de Sangue do HUCFF (1997), que encontrou em 8867 soros pesquisados a freqüência de 1,39\% de anti-HCV+, com o método EIA aplicado e repetido nos casos duvidosos.

$O$ teste $P C R$ realizado nas seis amostras reativas ao EIA e nas quatro reativas ao INNOLIA ${ }^{\circledR}$, somente em duas amostras foram confirmadas positivas pelo PCR. Em 32 amostras escolhidas aleatoriamente entre os resultados não-reativos ao EIA e ao INNO-LIA ${ }^{\circledR}$ não se encontroua presença de RNA-HCV pelo PCR

Para se comparar a prevalência de antiHCV na amostra estudada coma prevalência de anti-HCV na população de comparação de doadores de sangue (avaliada exclusivamente pelo EIA), calculou-se a razão de prevalência entre as duas subpopulações, encontrando um resultado de 4,75 (IC $95 \%=2,15$ a $10,5 \%$ e um valor $p=0,002$, pelo teste de Fisher) (Veja tabela 3).
Dois pacientes considerados reativos no teste de triagem e no teste suplementar INNO-LIA ${ }^{\circledR}$ não apresentaram, no momento do estudo, o RNA-HCV identificável no soro pelo método $P C R$, sendo o primeiro paciente reativo aos antígenos do core $\mathrm{Cl}$ e C2 e aos antígenos não-estruturais NSI, NS2, NS3, NS4. Já o segundo paciente apresentou forte reatividade somente a um único antígeno, o NS4.

Calculou-se a prevalência de hepatite C quando os três testes são aplicados em série; isto é, se nos 91 pacientes se aplicasse - EIA e depois, somente nos resultados positivos se testasse o INNO-LIA ${ }^{\oplus}$ e nestes reativos se confirmasse com o PCR. A prevalência de HCV+ obtida nos três testes aplicados em série foi de 2,2\% nesta amostra (com IC95\%=0,2-7,7\%) (Veja Figura I e Tabela 2).

A concordância entre os testes imunossorológicos foi alta (Coeficiente kappa =0,78, erro padrão=0,10, valor $p<0,002$ ). A ocorrência de resultados discordantes entre o EIA $3^{\text {a }}$ geração e o PCR foi de 10,5\%, e entre o INNO-LIA ${ }^{\circledR}$ e o PCR foi de 5,5\%.

\section{DISCUSSÃO}

Aprevalência de anti-HCV + no presente estudo foi de $6,6 \%$ nos 91 pacientes com LES testados com o EIA $3^{a}$ geração ( mesmo método empregado no Banco de Sangue do HUCFF). Comparando-a com a prevalência de anti-HCV + na população de doadores de sangue $(1,39 \%)$ encontramos uma su- 
Tabela 2 - Comparação de anti-HCV entre a população de doadores de sangue e pacientes com LES - RAZÃO DE PREVALÊNCIA (RP)

\begin{tabular}{lcccc}
\hline & Anti-HCV + & Anti-HCV & Total(n) & prevalência \\
& EIA $3^{\circ}$ & EIA $3^{\circ}$ & & $(\%)$ \\
LESt & 6 & 85 & 91 & 6,6 \\
Doadores & 123 & 8744 & 8867 & 1,39 \\
\hline \multicolumn{5}{c}{ Valorp $=0,002$} \\
& $\mathrm{RP}=4,75(2,15<\mathrm{p}<10,50)$ &
\end{tabular}

\section{Gráfico I - Prevalência de Hepatite $C$ no estudo de acordo com métodos imunossorológicos}

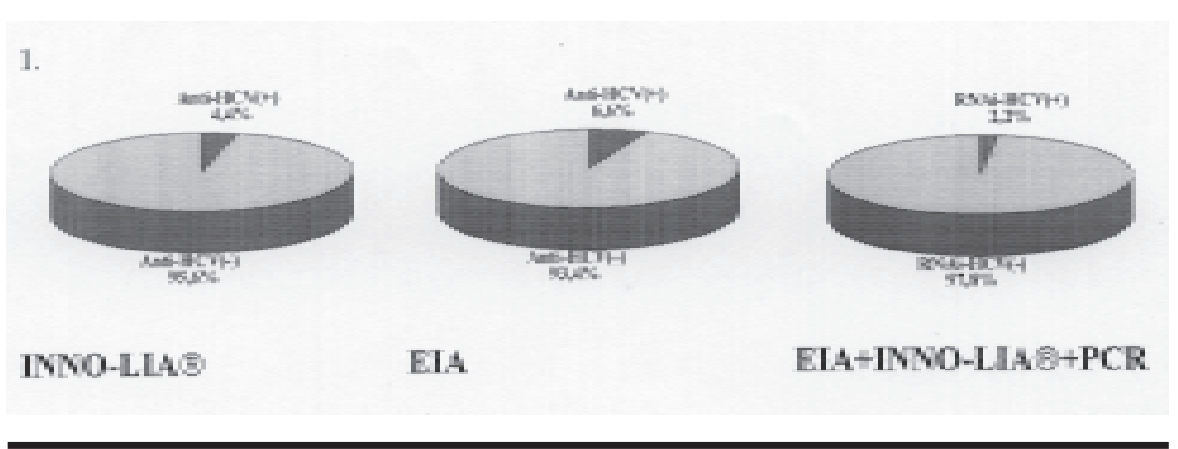

perioridade estatisticamente significativa da freqüência de anti-HCV nos pacientes com LES (Razão de Prevalência $=4,75$, com IC95\% $=2,15-10,5$ e valor $p<0,002$ ). Entretanto, identificou-se na amostra de pacientes com LES com uma probabilidade maior de exposição ao HCV que entre os doadores de sangue; isto é, na amostra estudada 15\% foram hemotransfundidos e $26 \%$ foram hospitalizados mais de uma vez ou foram submetidos a cirurgias de grande porte, ao passo que os doadores de sangue foram excluídos da seleção, na entrevista de triagem, sempre que apresentaram a presença de fatores de risco para aquisição do HCV.

Marchesoni et al. (1995) investigaram a associação entre hepatite C e LES excluindo da amostra os pacientes com LES comfatores de risco para hepatite $C$ e encontraram uma prevalência também ligeiramente superior ao encontrado na população de doadores de sangue $(6,6 \% \times 1 \%)$, embora sem significância estatística, sugerindo que a maior exposição ao HCV entre os pacientes com LES daamostra não parece ter influenciado de modo relevante os resultados deste estudo. Outros autores que investigaram estaassociação utilizando a população de doadores de sangue para comparação não encontraram diferença significativaentreas prevalências deanti-HCVnas amostras 1, 19,2,20,1,39.

Observaram-se resultados discordantes em dois pacientes, que foram positivos em ambos os testes imunossorológicos, mas negativos para a presença do RNAHCV pelo método PCR. O primeiro paciente foi reativo a todos seis antígenos pesquisados pelo INNO-LIA ${ }^{\circledR}$, e neste caso esses resultados não podem ser considerados falso-positivos, é necessário a repetição do PCR-RNA-HCV para se excluir a possi- bilidade infreqüente de uma viremia latente no soro analisado ou mesmo infecção passada; ao passo que o segundo paciente exibiu forte reatividade exclusivamente ao NS4, antígeno a qual pertence o epítopo Cl00-3, único reagente empregado nos testes EIA I'a geração, nos quais demonstrou-se uma alta freqüência de reações falso-positivas $11,17,21,40,42$.

Somente em dois pacientes não houve concordância entre os dois testes imunossorológicos testados. Os dois pacientes reativos ao EIA $3^{\text {a }}$ geração apresentaram resultados com uma leitura de densidade ótica próxima ao valor do cut off (superioridade inferior a 20\%) e não foram confirmados com o teste suplementar INNO$L I A^{\circledast}$. Apesar do alto coeficiente de concordância entre os testes (coef Kappa $=0,78$, erro padrão $=0,10$, valor $p<0,002$ ), ele é ainda inferior aos resultados encontrados em doadores de sangue, possivelmente devido aos indivíduos com LES apresentarem hiperglobulinemia e imunocomplexos circulantes ${ }^{11,17,20,21}$.

Um dos indivíduos com RNA-HCV apresentava o diagnóstico de LES provável durante 14 anos, caracterizado pela presença de poliartrite, leucopenia e FAN positivo. Manteve-se constante com estas manifestações clínico-laboratoriais, apesar da terapêutica com prednisona e cloroquina nos últimos meses. Não foi afastado o diagnóstico de crioglobulinemia mista e as manifestações auto-imunes da hepatite $C$ podem ser responsáveis pelo quadro clínico do paciente.

O outro indivíduo com RNA-HCV positivo preencheu os critérios diagnósticos de LES definido há 24 anos, com a presença de trombocitopenia, artrite, hipocomplementemia e anti-Sm positivo no soro. Observam-se fatores de risco para a aquisição do HCV, como hospitalizações e hemotransfusão na década de 80, período de alta incidência de hepatite pós-transfusional, ${ }^{3,41}$. 
Não houve evidências sugestivas de hepatopatia ao exame físico, e laboratorialmentefoi encontrado somente num único momento, há II anos, elevações discretas da ALT e AST 34. A corticoterapia, à qual a paciente foi submetida por longo período, não parece ter influenciado a infecção crônica pelo HCV.

\section{ConClusões}

A prevalência de hepatite $\mathrm{C}$ na amostra de pacientes com LES provável ou definido, utilizando-se um teste de triagem (EIA) foi significativamente maior do queadeuma população de doadores de sangue (6,6\% versus I,39\%). No entanto, considerando-se os resultados obtidos com testes confirmatórios INNOLIA ${ }^{\circledR}$ e PCR aplicados em série( prevalência de hepatite C de 2,2\%) parece não haver evidências de que pacientes com LES tenham maior risco de adquiriro HCV do que a populaçãa em geral, cuja prevalência tende a ser maior que a populaçãa de doadores de sangue.

Em pacientes com diagnóstico provável de LES, deve-se avaliar o custo/benefício de se investigar HCV e CME, já que as medidas terapêuticas para as referidas entidades clínicas podem significar mudança prognóstica favorável para essas pacientes.

Nos pacientes com LES e com testes para anti-HCV de $3^{a}$ geração positivos devese utilizar testes confirmatórios de biologia molecular para se avaliar a real prevalência de infecção pelo HCV.

\section{SUMMARY}

\section{Prevalence of Hepatitis $C$ in patients WITH Systemic Erythematosus Lupus of Hospital of Federal UniverSity of Rio de JANEIRO (HUCFF)}

BACKGROUND. Patients with systemic lupus erythematosus (SLE) are at risk for the acquisition of hepatitis $C$, owing to frequent hospitalization and hemotransfusion. Many clinicaland laboratorymanifestations related to auto-imunity are shared between Lupus and HCV infected patients.

OBIECTNE. To determine the prevalence of hepatitis $C$ in the population with SLE monitored in the out-patientservice of the special programs in-Collagenoses - of the University Hospital Clementino Fraga Filho (HUCFF-UFRI).

Methods. A Cross sectional study was performed, including 91 patients with the diagnosis of probable or definitive SLE (with 3 or more of the / / diagnostic criteria for SLE usedby the American College of Rheumatology - ACR) proceeding from the mentioned center between April/97 to September/97. Patients were evaluated on clinical and laboratory basis to determine activity of lupus disease (SLEDAI). All of patients were tested with UBIHCVEIA 4.0assayandINNO-LIAHCV Ablll confirmatory assay. PCR was performedonall EIA positive INNO-LIAHCVAbIII assay positive patients besides 32 patients negatives in both serological assays.

RESULTS. A significative increase in HCV prevalence among SLE patients was observed when compared with a control group of blood donors at HUCFF-UFR) (6.6 and $1.39 \%$, respectively; $p=0,02$ and confdence interval of $95 \%=5.5-13.8)$. However, if confirmative assays (INNO-LIA HCV ABIII and PCR ) are used, this difference can not be observed any more HCV prevalence of $2.2 \%$ with confidence interval of $95 \%=0.2-7.7$ ).

CONCLUSIONS. HCV prevalence using ELISA assay for screening showed a significant difference between SLE patients and a blood donors control group ( 6.6 and $1.39 \%$ respectively). However, if confirmatory assays are subsequently applied, no difference in HCV prevalence among both groups can be observed. Patients with a possible diagnosis of SLE should have a cost/ benefit analisys of testing for HCV. Rev Assoc Med Bras 2002; 48(I): 42-7]

KEY wORDS: Hepatitis C. Systemicc lupus erythematosus and auto-immunity.

\section{REFERÊNCIAS}

I. Abu-Shakra M, El-Sana S, Sikuler E, Neumann L, Buskila D. Hepatitis B and C viruses serology in patients with SLE. Lupus 1997; 6:543-4

2. Agnello V, Chung RT, Kaplan LM. A role for hepatitis $C$ virus infection in Type II yoglobulinemia. N Engl J Med 1992; 327:|490-5

3. Alter MJ. Epidemiology of hepatitis $C$ in the West. Semin Liver Dis 1995; 15:5-14.

4. Arif M, Al-Swayeh M, Al-Faleh FZ, Ramia S. Risk of hepatitis $C$ virus infection among household contacts of Saudi patients with chronic liver disease. J Viral Hepat 1996; 3:97-101.

5. Bombardier C, Gladman DD, Urowitz BM, Caron.D, Chang C. Derivation of the SLEDAl: a disease activity index for lupus patients. Arthritis Rheum 1992; 35:630-40.

6. Buskila D, Sikuler E, Shoenfeld Y. Hepatitis C virus, autoimmunity and rheumatic disease. Lupus 1997; 6:685-9.

7. Carneiro AJ, Costa CA e Coelho HS. 1994. Crioglobulina e VHC. In: Anais do XXXIII Congresso Brasileiro de Gastroenterologia [abstract]. Porto Alegre; 1994. p.73.

8. Casato M, Taliani G, Pucillo LP, Goffredo F, Lagana B, Bonomo L. Cryoglobulinemia and hepatitis C virus. Lancet 1991; 337: I047-8.

9. Choo QL, Kuo G, Weiner AJ, Overby LR, Bradley DV, Houghton M. Isolation of a cDNA clone derived from blood-borne non$A$, non-B viral hepatitis genome. Science 1989; 244:359-62.

10. Clifford BD, Donahue D, Smith L, Cable E, Luttig B, Manns M et al. High prevalence of serological markers of autoimmunity in patients with chronic hepatitis C. Hepatology 1995: 21:613-9.

I I. Craxi A, Valenza M, Fabiano C, Magrin S, Fiorentino G, Diquattro $O$. Third-generation hepatitis $C$ virus tests in assymptomatic antiHCV-positive blood donors. J Hepatol 1994: 21:730-4.

12. Epilnfo Version 6.03. A word processing, database and statistics program for public health on IBM-compatible microcomputers. Centers for Disease Control.

13. Garcia-Monforte A, Romero M, Diego A, Rivera J. Rheumatic and autoimmune manifestations in chronic hepatitis C virus infection. Arthritis Rheum 1995; 38 (Suppl 9):283.

14. Gladman D, Goldsmith CH, Urowitz MB, Bacon P, Bombardier C, Isenberg D. Sensibility to change of 3 systemic lupus erythematosus disease activity indices: international validation. I Rheumatol 1994; 21:|468-7|.

15. Hawker G, Gabriel S, Bombardier C , Goldsmith C, Caron D, Gladman D. A reliability of SLEDAI: a disease activity index for systemic lupus erythematosus. J Rheumatol 1993; 20:657-60.

16. Itoh K, Tanaka H, Shiga J, Hirakawa K, Akahane Y, Tsuda F et al. Hypocomplementemia associated with hepatitis $C$ viremia in sera from voluntary blood donors. Am J Gastroenterol 1994; 89:2019-23. 
17. Izopel J, Paven JL, Cazabat M, Dubois M, Thouvenot JP, Pascal JP et al. RIBA-3 or PCR as a complementary test to establish the diagnosis of $\mathrm{HCV}$ infection in a clincal virology laboratory. Hepatology 1995; 22 (4 pt II):358.

18. Jindal $R$, Solomon $M$ e Burrows L.. False positive tests for HIV in a women with lupus and renal failure. N Engl J Med 1994; 328:| $28 \mid-2$

19. Karakoc Y, Dilek K, Akalyn H, Gullulu M, Yavuz M, Ersoy A et al. Prevalence of hepatitis $C$ virus antibody in patients with systemic lupus erythematosus. Ann Rheum Dis 1997; 56:570-1.

20. Kowdley K, Subler D, Scheffel J, Moore B, Smith $H$. Hepatitis $C$ virus antibodies in systemic lupus erythematosus. I Clin Gastroenterol 1997; 25: 437-9.

21. Lavanchy D, Mayerat C, Morel B, Schneider P. Zufferey C, Gonvers || et al. Evaluation of third-generation assays for detection of antihepatitis $\mathrm{C}$ virus (HCV) antibodies and comparison with presence of HCV RNA in blood donors reactive to cl00-3 antigen. J Clin Microbiol 1994; 32:2272-5.

22. Liang M, Socher SA, Larson MG, Schur PH. Reliability and validity of six systems for the clinical assessment of disease activity in systemic lupus erythematosus. Arthritis Rheum 1989; 32:1107-17.

23. Lovy MR, Starkebaum G, Uberoi S. Hepatitis $C$ infection presenting with rheumatic manifestations: a mimic of rheumatoid arthritis. J Rheumatol 1996; 23:979-83.

24. Marcellin $P$, Descamps $V$, Martinot-Peignoux $M$, Larzul $D, X u L$, Boyer $N$ et al. Cryoglobulinaemia with vasculitis associated with hepatitis C virus infection. Gastroenterology 1993; 104:272-7.

25. Marchesoni A, Battafarano N, Podico M, Tosi S. Hepatitis C virus antibodies and systemic lupus erythematosus. Clin Exp Rheumatol 1995: 13: 267.

26. Misiani R, Bellavita P, Fenili D, Borelli G,
Marchesi D, Massazza M et al. Hepatitis C virus infection in patients with essential mixed cryoglobulinemia. Ann Intern Med 1992; I 17:573-7.

27. Moder KG e Lindor K. Musculoskeletal symptoms associated with hepatitis $C$. Arthritis Rheum 1995; 38 (Suppl 9): 283.

28. Naganine T, Ohtuka T, Takehara K Takayuki A Takagi $\mathrm{H}$, Masamoto $\mathrm{M}$ et al. Thrombocytopenia associated with hepatitis $C$ viral infection. J Hepatol 1996; 24:135-40.

29. Okaoue T, Sakamoto S, Itoh Y, Minami M, Yasui K, Sakamoto $M$ et al. Side effects of high-dose interferon therapy for chronic hepatitis C. J Hepatol 1996; 3:283-91.

30. Pascual M, Perrin L, Giostra E, Schifferli JA Hepatitis $C$ virus in patients with cryoglobulinaemia type II. J Infect Dis 1990; 162: 569-70.

31. Pawlotsky JM, Roudot-Thoraval F, Simmonds P. Mellor J. Ben Yahia MB, Andre C et al.. Extrahepatic immunologic manifestations in chronic hepatitis $C$ and hepatitis $C$ virus genotypes. Ann Intern Med 1995; 122:169-73.

32. Pawlotsky JM, Yahia MB, André C, Voisin MC, Intrator L, Roudot-Thoraval F et al. Immunological disorders in $\mathrm{C}$ virus chronic active hepatitis: a prospective case-control study. Hepatology 1994; 19:84I-8.

33. Perrot $S$, Job-Deslandre $C$, Puéchat $X$, Menkès $\mathrm{C}$. Serological study of HCV infection in 4 groups of cases of polyarthritis. Arthritis Rheum 1995; 38 (Suppl 9):283.

34. Petri M, Baker C, Goldman D. Liver function test (LFT) abnormalities in systemic lupus erythematosus (SLE). Arthritis Rheum 1993; 36(Suppl 9): S239.

35. Pujol FH, Ponce JG, Lema MG, Capriles F, Devesa $M$, Sirit $F$ et al. High incidence of Hepatitis $C$ virus in Hemodialysis patients in units with high prevalence. J Clin Microbiol 1996; 34:1633-6.

36. Quismorio F. Hematologic and limphoid abnormalities in systemic lupus erythe- matosus. In: Wallace DJ, Hahn BH, editors. Dubois' lupus erythematosus. $5^{\text {th }}$ ed. Baltimore: Willams \& Wilkins; 1997. p.793-8I6.

37. Arce Salinas A, Cardiel MH, Guzman J, Varela JA. Validity of retrospective disease activity assessment in systemic lupus erythematosus. J Rheumatol 1996; 23:846-9

38. Tan E, Cohen AS, Fries JF, Masi AT, Macshane D). Rothfield NF et al. The 1982 revised criteria for classification of systemic lupus erythematosus. Arthritis Rheum 1982; 25: | $27 \mid-7$.

39. Tanaka M, Kobayashi S, Tajima M, Hatta S, Ysobe $Y$, Hashimoto $H$. Hepatitis C virus $(\mathrm{HCV})$ antibodies in the rheumatic diseases. Clin Exp Rheumatol 1991; 9:308-9.

40. Theilmann L, Blazer M, Goeser T, GmelinK, Kommerell B, Fiehn W. False-positive anti$\mathrm{HCV}$ tests in rheumatoid arthritis. Lancet 1990; 335: 1346

4I. Tillmann H, Manns M. Mode of Hepatitis C virus infection, epidemiology, and chronicity rate in the general population and risk groups. Dig Dis Sci 1996; 4I(Suppl 1):27-40S.

42. Vitali C, Sciuto M, Neri R, Greco F, Mavridis AK, Tsioufas AG et al. Anti-hepatitis C virus antibodies in primary Sjögren' syndrome false positive results are relates to hypergama-globulinaemia. Clin Exp Rheumatol 1992: 10:103-9.

43. Wener WH, Johnson RJ, Sasso EH, Gretch DR. Hepatitis $C$ virus and rheumatic disease. Rheumatol 1996; 23:953-8.

44. Zignego AL, Ferri C, Giannini C, Monti M, La Civita I, Careccia G et al. Hepatitis C virus genotype analysis in patients with type II mixed cryoglobulinemia. Ann Intern Med 1996: 124: 31-4.

Artigo recebido: 05/04/200 I

Aceito para publicação: 23/08/200 I 